\title{
Second-order charge-density-wave transition in single crystals of $\mathrm{La}_{3} \mathrm{Co}_{4} \mathrm{Sn}_{13}$
}

\author{
Johannes Welsch, ${ }^{1}$ Sitaram Ramakrishnan, ${ }^{1}$ Claudio Eisele, ${ }^{1}$ Natalija van Welll, ${ }^{1}$ Andreas Schönleber $\odot,{ }^{1}$ \\ Sander van Smaalen $\odot,{ }^{1, *}$ Shidaling Matteppanavar, ${ }^{2}$ Arumugam Thamizhavel, ${ }^{2}$ Martin Tolkiehn, ${ }^{3}$ \\ Carsten Paulmann, ${ }^{4}$ and Srinivasan Ramakrishnan ${ }^{2, \dagger}$ \\ ${ }^{1}$ Laboratory of Crystallography, University of Bayreuth, 95447 Bayreuth, Germany \\ ${ }^{2}$ Department of Condensed Matter Physics and Materials Science, Tata Institute of Fundamental Research, Mumbai 400005, India \\ ${ }^{3}$ P24, PETRA III, DESY, 22607 Hamburg, Germany \\ ${ }^{4}$ Mineralogisch-Petrographisches Institut, Universität Hamburg, 20146 Hamburg, Germany
}

(Received 31 July 2019; revised manuscript received 27 November 2019; published 26 December 2019)

\begin{abstract}
We present the temperature dependence of the electrical resistivity, the magnetic susceptibility, and the specific heat of a high-quality single crystal of $\mathrm{La}_{3} \mathrm{Co}_{4} \mathrm{Sn}_{13}$. As opposed to earlier reports on this system, these bulk properties exhibit clear anomalies at the phase transition at $T^{*}=151(1) \mathrm{K}$, while the present data confirm the second-order character of this transition. X-ray diffraction with synchrotron radiation is used to solve the fourfold superstructure in space group $I 2_{1} 3$ as it exists below $T^{*}$ in the charge-density-wave (CDW) state of $\operatorname{La}_{3} \mathrm{Co}_{4} \mathrm{Sn}_{13}$. Unlike conventional CDW systems, we have observed hysteresis between the zero-field-cooled and field-cooled magnetization below the CDW transition. This discrepancy can be attributed to a possible magnetic instability arising out of correlations of Co in the lattice, developing at the CDW transition. The crystal structure shows that any modifications of the electronic state of Co might be due to modified binding characteristics of the Sn atoms comprising the trigonal prismatic coordination of $\mathrm{Co}$, while the coordination of Co itself is hardly changed at the phase transition. The superconducting transition is observed at $T_{\mathrm{sc}}=2.85(2) \mathrm{K}$. The superconducting energy gap is estimated as $5 \mathrm{~K}$ on the basis of the specific heat measured down to $0.1 \mathrm{~K}$. These results suggest that $\mathrm{La}_{3} \mathrm{Co}_{4} \mathrm{Sn}_{13}$ is a conventional weak-electron-phonon-coupling superconductor.
\end{abstract}

DOI: 10.1103/PhysRevMaterials.3.125003

\section{INTRODUCTION}

Superconducting stannides of the type $R_{3} \mathrm{~T}_{4} \mathrm{Sn}_{13}(R=$ $\mathrm{La}, \mathrm{Sr}, \mathrm{Ca}$ and $T=\mathrm{Co}, \mathrm{Rh}, \mathrm{Ir})$ were first reported by $\mathrm{Re}-$ meika et al. [1], Espinosa [2], and Hodeau et al. [3]. Similar compounds, like $\mathrm{Ce}_{3} \mathrm{Co}_{4} \mathrm{Sn}_{13}$ [4] and $\mathrm{Ce}_{3} \mathrm{Rh}_{4} \mathrm{Sn}_{13}$ [5], have been added to this list subsequently. Recently, this series has attracted attention due to the observation of structurally tuned quantum criticality in $\left(\mathrm{Ca}_{x} \mathrm{Sr}_{1-x}\right)_{3} \mathrm{Ir}_{4} \mathrm{Sn}_{13}$ and $\left(\mathrm{Ca}_{x} \mathrm{Sr}_{1-x}\right)_{3} \mathrm{Rh}_{4} \mathrm{Sn}_{13}$ [6-10]. In these series of compounds, the critical temperature $T^{*}$ of the structural transition decreases rapidly along with a concomitant increase in the superconducting transition temperature $T_{\mathrm{sc}}$, which peaks near the composition or pressure where $T^{*}$ extrapolates to $0 \mathrm{~K}$. The phase diagrams closely resemble those of many unconventional superconductors in the vicinity of magnetic quantum critical points. Such a competition or coexistence of multiple phase transitions is one of the frontiers of condensed matter physics.

$\mathrm{La}_{3} \mathrm{Co}_{4} \mathrm{Sn}_{13}$ is one of the compounds in the $\mathrm{R}_{3} \mathrm{~T}_{4} \mathrm{Sn}_{13}$ series of compounds, and it has also received a considerable amount of attention [11-14]. Unlike the other compounds of this series, the origin of the structural transition in $\mathrm{La}_{3} \mathrm{Co}_{4} \mathrm{Sn}_{13}$ and its low-temperature superstructure has remained controversial. Recent experiments did establish the second-order nature of the structural phase transition, but they failed to solve the crystal structure of the low-temperature

\footnotetext{
*smash@uni-bayreuth.de

†ramky@tifr.res.in
}

phase [15]. Another study has presented the low-temperature crystal structure of related $\mathrm{Ce}_{3} \mathrm{Co}_{4} \mathrm{Sn}_{13}$ [16].

In the present work, we provide strong evidence that the phase transition of $\mathrm{La}_{3} \mathrm{Co}_{4} \mathrm{Sn}_{13}$ at $T^{*} \approx 151 \mathrm{~K}$ is a second-order transition and we present a detailed model for the low-temperature superstructure. Electrical resistivity data, the specific heat, and magnetization data suggest that this phase transition is a charge-density-wave (CDW) transition. The temperature-dependent magnetic susceptibility reveals an unusual hysteresis at this transition between the zero-fieldcooled (ZFC) and field-cooled (FC) data. We also show that the superconductivity exhibited by $\mathrm{La}_{3} \mathrm{Co}_{4} \mathrm{Sn}_{13}$ is due to weak electron-phonon coupling by measuring the heat capacity down to $100 \mathrm{mK}$.

\section{EXPERIMENT}

\section{A. Synthesis and $x$-ray diffraction}

Single-crystalline samples of $\mathrm{La}_{3} \mathrm{Co}_{4} \mathrm{Sn}_{13}$ were grown using Sn flux. The purity of the elements, used in a stoichiometric ratio, was $99.999 \%$ for $\mathrm{La}$ and $\mathrm{Sn}$ and $99.99 \%$ for Co. The 3-4-13 stoichiometry of the samples was established by energy-dispersive x-ray analysis as measured in an electron microscope. The experimentally determined composition was $\mathrm{La}_{2.99(1)} \mathrm{Co}_{3.99(3)} \mathrm{Sn}_{13.02(2)}$, equal to the stoichiometry within standard uncertainties.

The single-crystalline nature of the sample was verified by Laue diffraction. The backreflection Laue diffraction method was also used to orient the single crystals along the lattice 
direction a. For transport and magnetization measurements, small oriented bars were cut by a wire saw from the large oriented crystal.

A small piece was cut off the large crystal of $\mathrm{La}_{3} \mathrm{Co}_{4} \mathrm{Sn}_{13}$ and then crushed in order to obtain single crystals of approximate dimensions of $0.1 \times 0.1 \times 0.1 \mathrm{~mm}^{3}$ for singlecrystal x-ray diffraction (SXRD) experiments. Single-crystal $\mathrm{X}$-ray diffraction was measured at station EH2 of beamline P24 of the PETRA-III extension at DESY in Hamburg, Germany, employing radiation with a wavelength of $\lambda_{\mathrm{P} 24}=$ $0.559421 \AA$. The temperature of the sample was regulated with a CRYOCOOL open-flow cryostat, employing helium as cryogas. Good data sets were obtained up to a resolution of $\left(\frac{\sin (\theta)}{\lambda}\right)_{\max }=0.7102 \AA^{-1}$ at temperatures of 25 and $180 \mathrm{~K}$, below and above the phase transition at $T^{*}$, respectively. Lattice parameters and integrated intensities of Bragg reflections were obtained by the software EVAL15 [17] and absorption correction was applied by the software SADABS [18]. Details of the data processing and of the structure refinements with the software JANA2006 [19] are given in the Supplemental Material [20].

The SXRD data at $180 \mathrm{~K}$ could successfully be indexed on a primitive cubic lattice with $a=9.6340(2) \AA$ (Table S2 in [20]). The crystal structure in space group $P m \overline{3} n$ gave an excellent fit to the SXRD with $R_{F}=0.028$. The resulting atomic parameters are equal to those reported for $\mathrm{La}_{3} \mathrm{Co}_{4} \mathrm{Sn}_{13}$ at room temperature [12] and they are close to those reported for other $\mathrm{R}_{3} \mathrm{~T}_{4} \mathrm{Sn}_{13}$ compounds (Table $\mathrm{S} 3$ in [20]) [16,21].

At $25 \mathrm{~K}$ the SXRD data of $\mathrm{La}_{3} \mathrm{Co}_{4} \mathrm{Sn}_{13}$ indicated a $2 a_{0} \times$ $2 a_{0} \times 2 a_{0}, \quad I$-centered supercell with $a_{0}=9.6167(2) \AA$, which is slightly smaller than the lattice parameter at $180 \mathrm{~K}$ (Table S2 in [20]). The superstructure in the cubic space group $I 2{ }_{1} 3$ gave a good fit to the SXRD data with $R_{F}=0.052$. The resulting superstructure is similar to that reported for $\mathrm{Ce}_{3} \mathrm{Co}_{4} \mathrm{Sn}_{13}$ (Table $\mathrm{S} 4$ in [20]) [16,21]. Other symmetries were tested, but they could not describe the SXRD data $[16,21]$.

\section{B. Electrical resistivity}

The electrical resistivity was measured along the cubic lattice direction of $\mathrm{La}_{3} \mathrm{Co}_{4} \mathrm{Sn}_{13}$, employing a bar-shape single crystal cut off the large, oriented as-grown single crystal (Sec. II A). Ohmic contacts were made by conductive silver paste and gold wires (40- $\mu \mathrm{m}$ diameter) for the standard fourprobe technique configuration. The temperature-dependent electrical resistivity was measured by employing a custombuilt setup involving an LR-700 (Linear Research, USA) AC bridge with a 5-mA current operating at a frequency of $16 \mathrm{~Hz}$. The resulting data are shown in Fig. 1. At low temperatures the electrical resistivity was measured in dependence on the magnetic field, demonstrating the field dependence of the superconducting phase transition (inset in Fig. 1). The anomaly at $T^{*}$ was not affected by a magnetic field, and a very weak magnetoresistance was observed for a magnetic field of $5 \mathrm{~T}$ and temperatures above $T_{\text {sc }}[20]$.

\section{Magnetic susceptibility}

The magnetic susceptibility of $\mathrm{La}_{3} \mathrm{Co}_{4} \mathrm{Sn}_{13}$ was measured with a commercial superconducting quantum interference de-

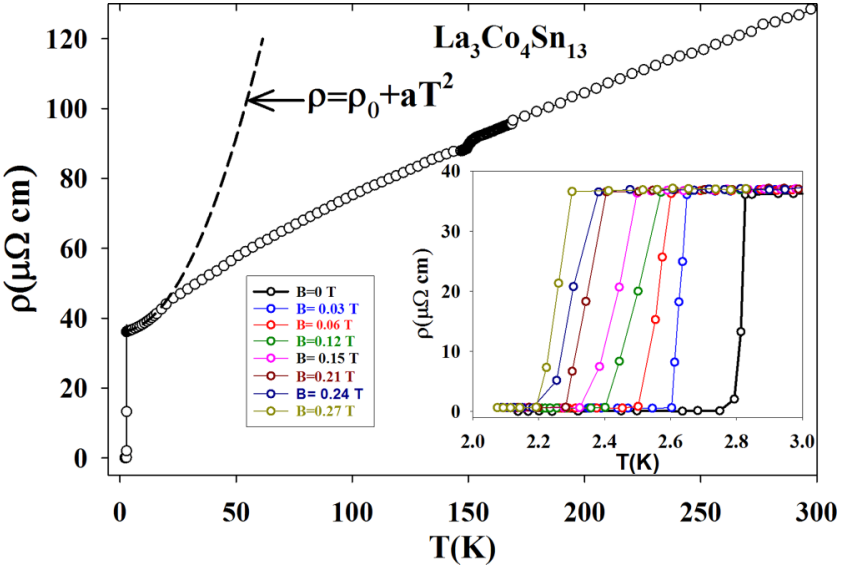

FIG. 1. Temperature dependence of the electrical resistivity $\rho(T)$ of $\mathrm{La}_{3} \mathrm{Co}_{4} \mathrm{Sn}_{13}$ as measured upon heating from 2 to $300 \mathrm{~K}$. An anomaly without thermal hysteresis is apparent around $T^{*}=$ 151(1) $\mathrm{K}$. The dotted line is a fit to the data for $3-20 \mathrm{~K}$, resulting in $\rho_{0}=38.17(1) \mu \Omega \mathrm{cm}$ and $A=0.022(2) \mu \Omega \mathrm{cm} / \mathrm{K}^{2}$ (see the discussion in the text). The crystal becomes superconducting below $2.85(2) \mathrm{K}$. The inset shows the magnetic field $(B)$ dependence of $\rho(T)$ around this transition.

vice magnetometer (Magnetic Properties Measurement System, MPMS 7, Quantum Design, USA). The temperature dependence of $\chi$ was obtained under ZFC and FC conditions for heating from 2 to $300 \mathrm{~K}$. The result is shown in Fig. 2.

\section{Specific heat}

The specific heat $C_{p}$ of a single crystal of $\mathrm{La}_{3} \mathrm{Co}_{4} \mathrm{Sn}_{13}$ was measured by the thermal relaxation method using a physical property measuring system (PPMS) (Quantum Design, USA) for heating from 2 to $270 \mathrm{~K}$ (Fig. 3). Data were also collected upon cooling down to $120 \mathrm{~K}$ (top inset of Fig. 3).

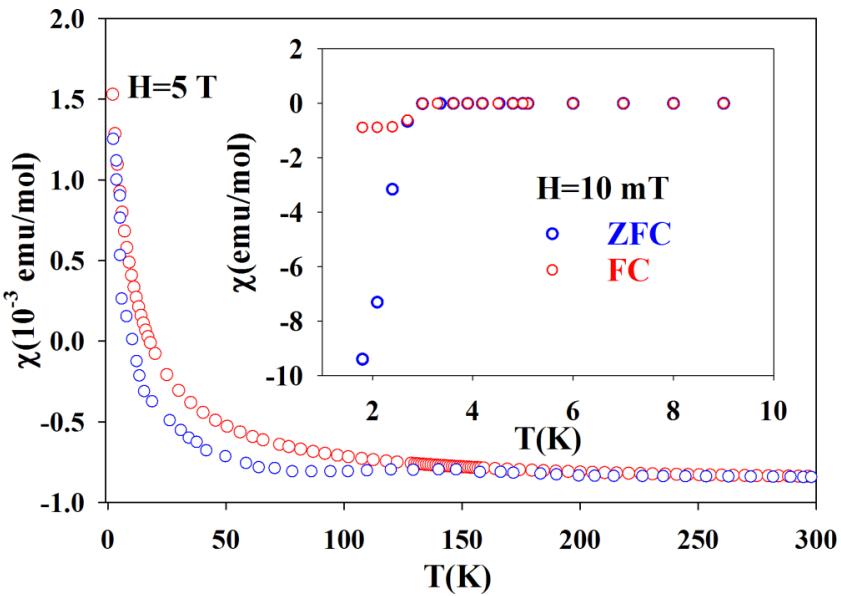

FIG. 2. Temperature dependence of the magnetic susceptibility $\chi(T)$ of $\mathrm{La}_{3} \mathrm{Co}_{4} \mathrm{Sn}_{13}$, as measured upon heating after ZFC and FC conditions in a field of $5 \mathrm{~T}$. The most prominent feature is the hysteresis between ZFC and FC conditions, starting at the temperature of transition $T^{*}=150(2) \mathrm{K}$. The inset displays the magnetic susceptibility at low temperatures around the superconducting transition at $2.85 \mathrm{~K}$ and measured in a field of $10 \mathrm{mT}$. 


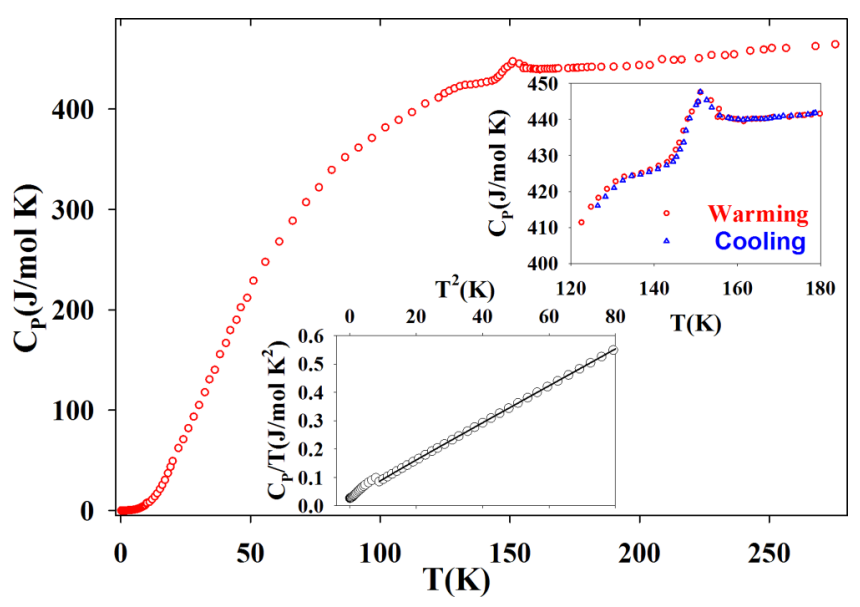

FIG. 3. Specific heat $C_{p}(T)$ of a single crystal of $\mathrm{La}_{3} \mathrm{Co}_{4} \mathrm{Sn}_{13}$ for $0.1-270 \mathrm{~K}$. Data were measured with the PPMS upon heating from 2 to $270 \mathrm{~K}$. The top inset provides an expanded view around the phase transition $T^{*}=150(2) \mathrm{K}$. The bottom inset shows $C_{p} / T$ vs $T^{2}$ for $0.1-9 \mathrm{~K}$ as measured with the custom-built setup. The solid line is a fit discussed in the text. An anomaly due to the superconducting phase transition is clearly visible at $T_{\mathrm{sc}}=2.8(1) \mathrm{K}$.

Furthermore, data were collected upon heating under application of a magnetic field of $5 \mathrm{~T}$. The $C_{p}(T)$ with and without a magnetic field are identical [20]. The specific heat at very low temperatures was measured in a custom-built system that employs a commercial dilution refrigerator (bottom inset of Fig. 3).

In a third experiment, differential scanning calorimetry (DSC) was measured upon heating and cooling within a temperature range encompassing the transition at $T^{*}$. The DSC data were calibrated, resulting in the temperature dependence of the specific heat. The change of the specific heat $\Delta C_{p}(T)$ was obtained after substraction of the background, including the lattice contribution. The change in entropy at the CDW transition was computed by integration of $\Delta C_{p} / T$. The data are shown in Fig. 4.

\section{DISCUSSION}

Single crystals of $\mathrm{La}_{3} \mathrm{Co}_{4} \mathrm{Sn}_{13}$ were grown using $\mathrm{Sn}$ flux. The phase transition at $T^{*}$ is reflected by anomalies at $T^{*}=$ 151(1) $\mathrm{K}$ in the temperature dependences of the electrical resistivity $\rho(T)$, the specific heat $C_{p}(T)$, and the magnetic susceptibility $\chi(T)$ (Figs. 1-3). This value agrees well with the recently reported value of $150 \mathrm{~K}$ [15]. Hysteresis is not observed, signifying a second-order phase transition at $T^{*}$.

The excellent quality of our single crystal can be derived from the behavior of $\rho(T)$, with the anomaly at $T^{*}$ being much more pronounced in the present data than in published data [15,22]. Furthermore, the superconducting transition takes place at $T_{\mathrm{sc}}=2.85(2) \mathrm{K}$, matching the highest published value for this transition [12]. The present value for $T_{\mathrm{sc}}$ is significantly higher than the value of $2 \mathrm{~K}$ as obtained in [22], which also reported the absence of an anomaly in $\rho(T)$ at $T^{*}$.

The continuous character of the structural transition is confirmed by the change of the specific heat due to the phase transition $\Delta C_{p}(T)$ as it has been derived from calibrated

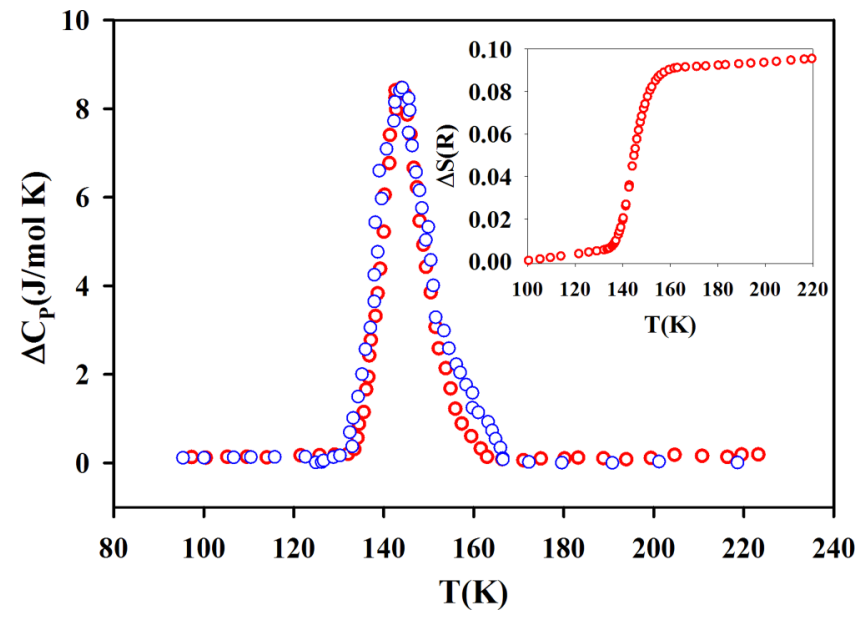

FIG. 4. Temperature dependence of the change in specific heat of $\mathrm{La}_{3} \mathrm{Co}_{4} \mathrm{Sn}_{13}$, as derived from DSC measured on heating (red) and on cooling (blue). The inset shows the change in entropy near the phase transition.

DSC measurements. Data obtained upon heating and data obtained upon cooling the sample exhibit coinciding peaks in $\Delta C_{p}(T)$ (Fig. 4). The structural transition leads to an increase in the entropy of $\Delta S=0.1 R$, where $R$ is the gas constant (Fig. 4). This change of entropy and the maximum change in specific heat of $\left[\Delta C_{p}(T)\right]_{\max }=8.6 \mathrm{~J} / \mathrm{mol} \mathrm{K}$ at $T^{*}=151 \mathrm{~K}$ are comparable to the corresponding values of conventional CDW systems such as $\left[\Delta C_{p}(T)\right]_{\max }=8 \mathrm{~J} / \mathrm{mol} \mathrm{K}$ and $\Delta S=$ $0.18 R$ for $\mathrm{K}_{0.3} \mathrm{MoO}_{3}[23-25]$ and $\left[\Delta C_{p}(T)\right]_{\max } \approx 9 \mathrm{~J} / \mathrm{mol} \mathrm{K}$ and $\Delta S=0.08 R$ for $\mathrm{NbSe}_{3}$ [26]. Accordingly, the small value of $\left[\Delta C_{p}(T)\right]_{\max }$ and the absence of hysteresis are features expected for a second-order CDW transition [27].

A second anomaly is present in $C_{p}(T)$ at the superconducting transition. The temperature dependence between 3 and $20 \mathrm{~K}$ (above $T_{\mathrm{sc}}$ ) could be fitted to $C_{p}(T)=\gamma T+\beta T^{3}$, resulting in a Sommerfeld coefficient of moderate magnitude $\gamma=30 \mathrm{~mJ} / \mathrm{mol} \mathrm{K}{ }^{2}$ and a Debye temperature of $\theta_{D}=179 \mathrm{~K}$ (bottom inset of Fig. 3). The specific heat within the superconducting state $(0.1-2.8 \mathrm{~K})$ could be fitted to $C_{p} / T=\gamma+$ $\beta T^{2}+C T \exp [-\Delta(0) / T]$, with $C=0.180(4) \mathrm{mJ} / \mathrm{mol} \mathrm{K}{ }^{3}$. The resulting value of $\Delta(0)=5 \mathrm{~K}$ leads to $2 \Delta / k_{B} T_{\mathrm{sc}}=3.57$, which is close to the theoretical value of 3.52 in BardeenCooper-Schrieffer (BCS) theory [28], and thus suggests that the superconductivity is phonon mediated BCS type. The character of the anomaly at $T_{\mathrm{sc}}$ is typical for a superconducting transition. This implies that the CDW continues to exist within the superconducting state because otherwise the entropy lost at the CDW transition (inset in Fig. 4) should have been restored at the superconducting transition.

The most interesting feature of the temperature-dependent magnetic susceptibility is the hysteresis observed between $\mathrm{ZFC}$ and $\mathrm{FC}$ data between $2 \mathrm{~K}$ and $T^{*}=151 \mathrm{~K}$ (Fig. 2). Many systems with CDW ordering do not show any difference between ZFC and FC magnetization across the phase transition. Although $\mathrm{La}_{3} \mathrm{Co}_{4} \mathrm{Sn}_{13}$ is nonmagnetic, there is a significant contribution from the $3 d$ electrons of Co to the density of states at the Fermi level, as deduced by the band structure calculations and photoemission studies [29,30]. This 


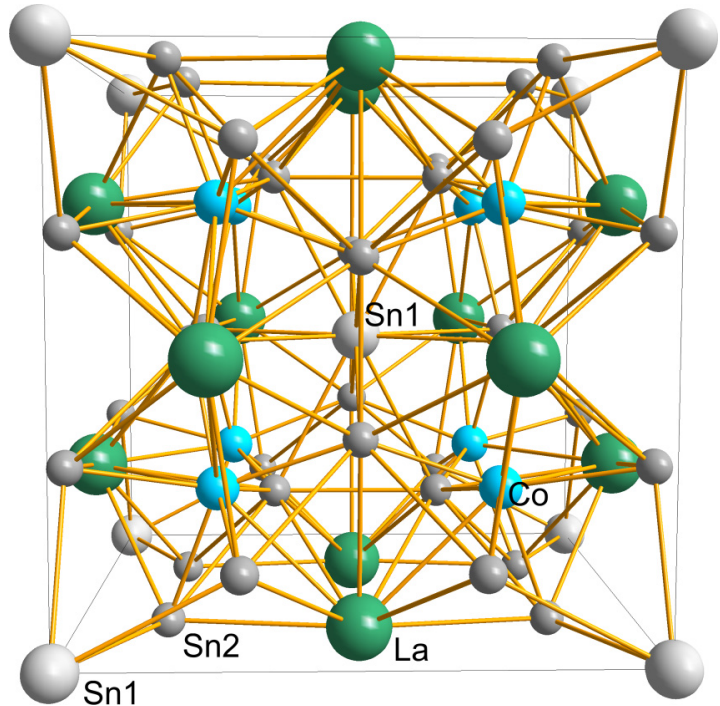

FIG. 5. Perspective along a of one unit cell of the crystal structure of $\mathrm{La}_{3} \mathrm{Co}_{4} \mathrm{Sn}_{13}$ at $180 \mathrm{~K}$. Small blue circles represent Co atoms, large green circles are La, light gray circles of intermediate sizes are $\mathrm{Sn} 1$ atoms located at the origin (corners of the unit cell) and exactly in the middle of the unit cell, and small circles of darker gray are Sn2.

suggests that different contributions of the Co $3 d$ electrons to the band structures of the high-temperature and superstructure phases may be responsible for the different magnetic behavior of these phases.

Above $T^{*}$ the crystal structure of $\mathrm{La}_{3} \mathrm{Co}_{4} \mathrm{Sn}_{13}$ is cubic $P m \overline{3} n$ with $a=9.6340$ (2) $\AA$ at $T=180 \mathrm{~K}$ (Fig. 5). This crystal structure comprises four crystallographically independent atoms, designated La1, Sn1, Sn2, and Co1 in [20]. It has been characterized by the atomic environments, which are an icosahedral coordination of $\mathrm{Sn} 1$ by twelve $\mathrm{Sn} 2$ atoms at equal distances of $3.277 \AA$, a cuboctahedral coordination of the La atom by four $\operatorname{Sn} 2$ atoms at $3.344 \AA$ and eight $\operatorname{Sn} 2$ atoms at a distance of $3.425 \AA$, and trigonal prismatic coordination of Co1 by six $\mathrm{Sn} 2$ at equal distances of $2.618 \AA$ [20,21]. Not mentioned in [21] was the $(12+2)$-fold irregular coordination of $\mathrm{Sn} 2$ by three La1 atoms, one $\mathrm{Sn} 1$ atom, and eight $\mathrm{Sn} 2$ atoms at distances between 3.02 and $3.82 \AA$, supplemented by two Co atoms at very short distances of $2.618 \AA$. The crystal structure can be considered as a heavily distorted cubic closed packing (ccp) of Sn and La atoms with Co1 in one-quarter of the octahedral sites. The distortion from ccp towards $P m \overline{3} n$ involves major displacements of the $\mathrm{La}$ and $\mathrm{Sn} 2$ atoms while keeping the connectivity of the atoms (12-fold coordination of each atom of the ccp).

The highly irregular environment of $\mathrm{Sn} 2$ in the basic structure makes it difficult to find an unequivocal structural signature of the CDW formation. It has been proposed that variations of the environment of $\mathrm{Co} 1$ are at the origin of the CDW [11,16,31]. Variations of the $\mathrm{Co} 1(\mathrm{Sn} 2)_{6}$ coordination polyhedra are supposed to affect the otherwise strong hybridization between $\mathrm{Sn} 25 p$ and Co $3 d$ states [15] and thus influence the properties. Here we find that the coordination of Co1 becomes only marginally irregular within the CDW superstructure, with six $\mathrm{Sn} 2$ atoms at different distances with a variation of $\Delta d=0.085 \AA$ for Cola and Colb and a smaller variation of $\Delta d=0.046 \AA$ for Colc and Cold (Table S8 in [20]). On the other hand, the irregular environment of the single $\mathrm{Sn} 2$ atom at $180 \mathrm{~K}(\Delta d=0.796 \AA)$ becomes eight different environments with four $\mathrm{Sn} 2$-type atoms with $\Delta d \approx 0.8 \AA$, two Sn2-type atoms with $\Delta d=1.2 \AA$, and two Sn2-type atoms with $\Delta d=1.7 \AA$ (Table S8 in [20]). The irregularity of the $\mathrm{Sn} 2$ environment is clearly larger in the CDW state than in the normal state. This enhanced irregularity might be at the origin of the CDW formation. For example, the modified bonding characteristics of these Sn2 atoms may affect the $p-d$ hybridization with cobalt, although the $\mathrm{Co} 1(\mathrm{Sn} 2)_{6}$ coordination polyhedra themselves are hardly affected by CDW formation. Band structure calculations of the superstructure, which might shed light on these questions, in particular the magnetic properties of the CDW phase, are beyond the scope of the present work.

\section{CONCLUSION}

We have presented the temperature-dependent electrical resistivity, magnetization, and specific heat of a high-quality single crystal of $\mathrm{La}_{3} \mathrm{Co}_{4} \mathrm{Sn}_{13}$. Furthermore, we have employed synchrotron radiation for crystal structure determinations of the normal and CDW states. Unlike previous measurements, we observed a clear anomaly in the electrical resistivity and magnetization. More importantly, the synchrotron measurements showed a continuous phase transition from a cubic to another cubic structure with a concomitant CDW transition. We proposed that a possible modified $p$ - $d$ hybridization between Co and $\mathrm{Sn} 2$ atoms within the CDW state is caused by strong distortions of the $\mathrm{Sn} 2$ environments. Those distortions will lead to modified bonding characteristics of $\mathrm{Sn} 2$, including the bonds towards $\mathrm{Co}$, while the geometry of the $\operatorname{Co}(\operatorname{Sn} 2)_{6}$ coordination polyhedra is hardly affected.

Previous studies [15] which included band structure calculations estimated the Sommerfeld coefficient $\gamma_{t h}$ to be $17 \mathrm{~mJ} / \mathrm{mol} \mathrm{K}^{2}$, which gives an enhancement factor of 1.76 since the experimental value of $\gamma$ is $30 \mathrm{~mJ} / \mathrm{mol} \mathrm{K}^{2}$. We believe that such an enhancement arises probably due to electron-phonon coupling similar to the one observed in A-15 compounds [32]. The superconductivity observed in $\mathrm{La}_{3} \mathrm{Co}_{4} \mathrm{Sn}_{13}$ can be understood in terms of the conventional BCS theory with a energy gap value of $5 \mathrm{~K}$ from the heat capacity data between 2.8 and $0.1 \mathrm{~K}$.

\section{ACKNOWLEDGMENTS}

We thank D. Buddikot for his help in performing the heat capacity measurements. We thank Leonardo Alves Bigão for his help with initial x-ray-diffraction experiments. This research was funded by the Deutsche Forschungsgemeinschaft (DFG, German Research Foundation) Grant No. 265092781 and by the Alexander-von-Humboldt Foundation within their research-group linkage program. 
[1] J. P. Remeika, G. P. Espinosa, A. S. Cooper, H. Barz, J. M. Rowell, D. B. McWhan, J. M. Vandenberg, D. E. Moncton, Z. Fisk, L. D. Woolf, H. C. Hamaker, M. B. Maple, G. Shirane, and W. Thomlinson, Solid State Commun. 34, 923 (1980).

[2] G. P. Espinosa, Mater. Res. Bull. 15, 791 (1980).

[3] J. L. Hodeau, M. Marezio, J. P. Remeika, and C. H. Chen, Solid State Commun. 42, 97 (1982).

[4] C. Israel, E. M. Bittar, O. E. Aguero, R. R. Urbano, C. Rettori, I. Torriani, P. G. Pagliuso, N. O. Moreno, J. D. Thompson, M. F. Hundley, J. L. Sarrao, and H. A. Borges, Physica B 359-361, 251 (2005).

[5] U. Köhler, A. Pikul, N. Oeschler, T. Westerkamp, A. M. Strydom, and F. Steglich, J. Phys.: Condens. Matter 19, 386207 (2005).

[6] L. E. Klintberg, S. K. Goh, P. L. Alireza, P. J. Saines, D. A. Tompsett, P. W. Logg, J. Yang, B. Chen, K. Yoshimura, and F. M. Grosche, Phys. Rev. Lett. 109, 237008 (2012).

[7] S. K. Goh, D. A. Tompsett, P. J. Saines, H. C. Chang, T. Matsumoto, M. Imai, K. Yoshimura, and F. M. Grosche, Phys. Rev. Lett. 114, 097002 (2015).

[8] W. C. Yu, Y. W. Cheung, P. J. Saines, M. Imai, T. Matsumoto, C. Michioka, K. Yoshimura, and S. K. Goh, Phys. Rev. Lett. 115, 207003 (2015).

[9] C. N. Kuo, H. F. Liu, C. S. Lue, L. M. Wang, C. C. Chen, and Y. K. Kuo, Phys. Rev. B 89, 094520 (2014).

[10] C. N. Kuo, C. W. Tseng, C. M. Wang, C. Y. Wang, Y. R. Chen, L. M. Wang, C. F. Lin, K. K. Wu, Y. K. Kuo, and C. S. Lue, Phys. Rev. B 91, 165141 (2015).

[11] A. Slebarski and J. Goraus, Phys. Rev. B 88, 155122 (2013).

[12] E. L. Thomas, H.-O. Lee, A. N. Bankston, S. MaQuilon, P. Klavins, M. Moldovan, D. P. Young, Z. Fisk, and J. Y. Chan, J. Solid State Chem. 179, 1642 (2006).

[13] A. Slebarski, M. M. Maska, M. Fijałkowski, C. A. McElroy, and M. B. Maple, J. Alloys Compd. 642, 866 (2015).

[14] P. Neha, P. Srivastava, R. Jha, Shruti, V. P. S. Awana, and S. Patnaik, J. Alloys Compd. 665, 333 (2016).

[15] Y. W. Cheung, J. Z. Zhang, J. Y. Zhu, W. C. Yu, Y. J. Hu, D. G. Wang, Y. Otomo, K. Iwasa, K. Kaneko, M. Imai, H. Kanagawa, K. Yoshimura, and S. K. Goh, Phys. Rev. B 93, 241112(R) (2016).
[16] Y. Otomo, K. Iwasa, K. Suyama, K. Tomiyasu, H. Sagayama, R. Sagayama, H. Nakao, R. Kumai, and Y. Murakami, Phys. Rev. B 94, 075109 (2016).

[17] A. M. M. Schreurs, X. Xian, and L. M. J. Kroon-Batenburg, J. Appl. Crystallogr. 43, 70 (2010).

[18] G. M. Sheldrick, SADABS, version 2008/1 (University of Göttingen, Göttingen, 2008).

[19] V. Petříček, M. Dušek, and L. Palatinus, Z. Kristallogr. Cryst. Mater. 229, 345 (2014).

[20] See Supplemental Material at http://link.aps.org/supplemental/ 10.1103/PhysRevMaterials.3.125003 for details on the diffraction experiments and values of the structural parameters at 25 and $180 \mathrm{~K}$, as well as the electrical resistivity and specific heat measured in a magnetic field.

[21] J. L. Hodeau, J. Chenavas, M. Marezio, and J. P. Remeika, Solid State Commun. 36, 839 (1980)

[22] A. Slebarski, M. Fijalkowski, M. M. Maska, M. Mierzejewski, B. D. White, and M. B. Maple, Phys. Rev. B 89, 125111 (2014).

[23] J. W. Brill, M. Chung, Y.-K. Kuo, X. Zhan, E. Figueroa, and G. Mozurkewich, Phys. Rev. Lett. 74, 1182 (1995).

[24] R. S. Kwok and S. E. Brown, Phys. Rev. Lett. 63, 895 (1989).

[25] R. S. Kwok, G. Gruner, and S. E. Brown, Phys. Rev. Lett. 65, 365 (1990).

[26] S. Tomić, D. D. K. Biljaković, J. Cooper, P. Monceau, and A. Meerschaut, Solid State Commun. 38, 109 (1981).

[27] H. F. Liu, C. N. Kuo, C. S. Lue, K. Z. Syu, and Y. K. Kuo, Phys. Rev. B 88, 115113 (2013).

[28] J. Bardeen, L. N. Cooper, and J. R. Schrieffer, Phys. Rev. 108, 1175 (1957).

[29] W. J. Ban, J. L. Luo, and N. L. Wang, J. Phys.: Condens. Matter. 29, 405603 (2017).

[30] A. Slebarski, J. Goraus, P. Witas, L. Kalinowski, and M. Fijalkowski, Phys. Rev. B 91, 035101 (2015).

[31] K. Suyama, K. Iwasa, Y. Otomo, K. Tomiyasu, H. Sagayama, R. Sagayama, H. Nakao, R. Kumai, Y. Kitajima, F. Damay, J.-M. Mignot, A. Yamada, T. D. Matsuda, and Y. Aoki, Phys. Rev. B 97, 235138 (2018).

[32] G. W. Webb, Z. Fisk, J. J. Engelhardt, and S. D. Bader, Phys. Rev. B 15, 2624 (1977). 